INPLASY

PROTOCOL

To cite: Gao et al. Curative Effects of Traditional Chinese medicine on Liver Fibrosis: a protocol for a systematic review and meta-analysis. Inplasy protocol 202110017. doi:

10.37766/inplasy2021.1.0017

Received: 5 January 2021

Published: 5 January 2021

Corresponding author:

Xia Gao

gaoxia198643@163.com

Author Affiliation:

Chongqing Medical University

Support: NSFC.

Review Stage at time of this submission: The review has not yet started.

Conflicts of interest: None declared.

\section{Curative Effects of Traditional Chinese medicine on Liver Fibrosis: a protocol for a systematic review and meta-analysis}

Gao, X1; Cao, W2.

Review question / Objective: Assessing the effectiveness and safety of Traditional Chinese medicine on liver fibrosis is the main purpose of this systematic review protocol.

Condition being studied: Chronic liver disease will contribute to liver fibrosis, which features replacement of liver tissue by fibrosis, scar tissue, and regenerative nodules, leading to liver dysfunction. This will lead to impairment of liver and affect patients' quality of life. Thus, the prevention and treatment of liver fibrosis is significant. Nowadays, there is no specific treatment, despite of early intervention or control of etiologies and hepatic inflammation and regulation of hepatic extracellular matrix (ECM) metabolism and stellate cell (HSC) activation. It has been reported that traditional Chinese medicine could be effective to the liver fibrosis.

INPLASY registration number: This protocol was registered with the International Platform of Registered Systematic Review and Meta-Analysis Protocols (INPLASY) on 5 January 2021 and was last updated on 5 January 2021 (registration number INPLASY202110017).

\section{INTRODUCTION}

Review question / Objective: Assessing the effectiveness and safety of Traditional Chinese medicine on liver fibrosis is the main purpose of this systematic review protocol.
Condition being studied: Chronic liver disease will contribute to liver fibrosis, which features replacement of liver tissue by fibrosis, scar tissue, and regenerative nodules, leading to liver dysfunction. This will lead to impairment of liver and affect patients' quality of life. Thus, the prevention 
and treatment of liver fibrosis is significant. Nowadays, there is no specific treatment, despite of early intervention or control of etiologies and hepatic inflammation and regulation of hepatic extracellular matrix (ECM) metabolism and stellate cell (HSC) activation. It has been reported that traditional Chinese medicine could be effective to the liver fibrosis.

\section{METHODS}

Participant or population: Participants who were 18 years or older with liver fibrosis will be included in spite of the gender, race, education or economic status.

Intervention: Experimental interventions include Traditional Chinese medicine therapy.

Comparator: Control interventions would be western medicine therapy.

Study designs to be included: All randomised controlled trials(RCTs) about traditional Chinese medicine on liver fibrosis which were reported in English and Chinese will be included. Trials with twoarm or three-arm parallel design will be also included. Non-RCTs, quasi-RCTs, case series, reviews, animal studies and any study with a sample size of less than ten participants will be excluded.

Eligibility criteria: The titles and abstracts of all searched studies will be reviewed and screened independently by two reviewers, aiming at identifying eligible trials and eliminating duplicated or irrelevant studies in line with the criteria; the full text of all possibly eligible studies will obtained if necessary. A discussion with the third reviewer is planned to solve the disagreements. A PRISMA-P flow diagram will be used to show the study selection process.

Information sources: The search strategy for PubMed is shown in table 1. The following search keywords will be used: Traditional Chinese medicine (e.g., "Chinese Drugs, Plant" or "Chinese Herbal Drugs" or "Herbal Drugs, Chinese" or
"Plant Extracts, Chinese" or "Chinese Plant Extracts" or "Extracts, Chinese Plant "); Liver Fibrosis. (e.g., "Liver Cirrhosis" or "Liver Cirrhosis, Biliary" or "Liver Cirrhosis, Alcoholic" ); randomized controlled trial (e.g., "randomized controlled trial" or "controlled clinical trial" or "random allocation" or "randomized" or "randomly" or "double-blind method" or "single-blind method" or "clinical trial". The equivalent search keywords will be used in the Chinese databases.

Main outcome(s): The primary outcome will be the levels of serum hyaluronic acid (HA), laminin (LN), type III procollagen (PC-III), and type IV procollagen (IV-C). There are no secondary outcomes.

Quality assessment / Risk of bias analysis: Two independent reviewers will access the quality of included literature and complete the Standards for Reporting Interventions in Clinical Trials of Acupuncture(STRICTA) checklist with the Cochrane collaboration risk-of-bias assessment method.

Strategy of data synthesis: RevMan V.53 will be used for data synthesis. The level of statistical heterogeneity will determine how the data will be synthesised and analysed. The random-effects model will be used if the 12 value is no less than $50 \%$. The fixedeffects model will be used if the heterogeneity tests show little statistical heterogeneity. If there is meaningful heterogeneity that cannot be explained by any assessment, meta-analysis will not be performed. If necessary, each subgroup will be carefully considered for subgroup analysis.

Subgroup analysis: Subgroup analysis will be conducted if the data are sufficient, according to the factors different outcomes and different control interventions.

Sensibility analysis: Sensitivity analysis will be conducted to test the robustness of the review conclusions if possible. The impacts of sample size, study design, methodological quality, and missing data will be evaluated. 
Country(ies) involved: China.

Keywords: systematic review, traditional

Chinese medicine, liver fibrosis.

Contributions of each author:

Author 1 - Xia Gao.

Email: gaoxia198643@163.com

Author 2 - Wenfu Cao.

Email: caowenfu9316@163.com 